\title{
Hábitos alimenticios y actividad física en los escolares de cuarto a sexto grado de nivel primaria de dos instituciones educativas públicas de Lima Este, 2015
}

\author{
Alimentary habits and physical activity in school children from fourth to sixth grade primary level of \\ two public educational institutions in East Lima, 2015
}

Naara Andrea Hernández Oblitas ${ }^{*}$, Lila Jaramillo Cano $^{2}$

\section{RESUMEN}

Objetivo: Determinar si exiten diferencias en los hábitos alimentarios y actividad física en los escolares de cuarto a sexto grado del nivel primario de dos instituciones educativas públicas de Lima Este, 2015. Material y métodos: De diseño no experimental, de corte transversal, de tipo descriptivo - comparativo. Los participantes fueron 571 escolares de 9 a 12 años. El muestreo fue no probabilístico de tipo censal. Se utilizó el test rápido de hábitos alimentarios Krece Plus y test corto de actividad física Krece Plus, validado por Serra y Cols (2003). Resultados: En la Institución María Goretti el 77.7\% (202) de los escolares tienen hábitos alimentarios alto y en la Institución 5 de abril sólo es el 70.4\% (219). En los hábitos alimentarios según género y lugar de procedencia no hay diferencias significativas en ambas instituciones $(\mathrm{p}>, 05)$. Asimismo en cuanto a la actividad física en la Institución 5 de Abril el 51.1\% (159) es mala y en la Institución María Goretti, el 68.5\% (178) es regular. En la actividad física según el género no hay diferencias significativas entre ambas instituciones $(\mathrm{p}>, 05)$. No obstante, en la institución 5 de abril según el lugar de procedencia no hay diferencias significativas $(\mathrm{p}>, 05)$, sin embargo se encontró diferencia significativa en la institución María Goretti $(\mathrm{p}<, 05)$. Conclusión: Existe diferencias significativas de los hábitos alimentarios y la actividad física en los escolares de ambas instituciones $(\mathrm{p}<.05)$.

Palabras clave: Hábitos alimenticios, actividad física, escolares, estilo de vida, promoción de la salud.

\begin{abstract}
Objective: Determine if there are differences Alimentary habits and physical activity in school children from fourth to sixth grade primary level of two public educational institutions in East Lima, 2015. Material and methods: design: non-experimental, cross-sectional, descriptive - comparative. The participants were school children from 9 to 12 years. The sampling was non probabilistic census type. The quick test of alimentary habits and the short test of physical activity by Krece Plus were used, validated by Serra and Cols. (2003). Results: in the Maria Goretti institution the $77.7 \%$ (202) of school children have alimentary habits high and in te " 5 de Abril” institution only the $70.4 \%$ (219). In alimentary habits according to gender and place of origin, there are no significant differences in both institutions $(\mathrm{p}>, 05)$. Also according to physical activity in the " 5 de Abril" institution 51.1\% (159) is bad and in the Maria Goretti institution, 68.5\% (178) is regular. In te Physical activity according to gender, there are not significant differences between the two institutions ( $>>, 05)$. Nevertheless, in the " 5 de Abril" institution, according to place of origin there is not significant differences ( $>>, 05)$, however significant difference was found in the institution María Goretti $(\mathrm{p}<, 05)$. Results: in the Maria Goretti institution the $77.7 \%$ (202) of school children have high alimentary habits and in the " 5 de abril" institution only the $70.4 \%$ (219). Conclusions: there are significant differences of alimentary habits and physical activity in school children of both institutions $(\mathrm{p}<, .05)$.
\end{abstract}

Keywords: physical activity, school children, lifestyle, alimentary habits, health promotion.

${ }^{1}$ Licenciada en Enfermería, Clínica Good Hope, Miraflores, Lima, Perú.

${ }^{2}$ Licenciada en Enfermería, Clínica Good Hope, Miraflores, Lima, Perú. 


\section{INTRODUCCIÓN}

En las últimas décadas se han mostrado evidencias sobre la importancia de una buena alimentación, especialmente en las etapas de desarrollo. Asimismo la práctica de buenos hábitos alimenticios y actividad física durante la niñez, siendo fundamental para alcanzar el máximo desarrollo físico e intelectual del escolar (Zegarra, Olivares-Atahualpa, Solano-Isidro, \& Musablon-Oyitas, 2011).

Los escolares están en una etapa de crecimiento con modificaciones morfológicas y fisiológicas, por ello se le considera un periodo de riesgo y, por lo tanto, resulta esencial valorar los hábitos de alimentación y actividad física para poder identificar los problemas de salud más frecuentes (Macias, Gordillo, \& Camacho, 2012).

Sin embargo, el crecimiento y desarrollo normal de los niños, están altamente influenciados por los hábitos alimenticios y la actividad física que éstos presentan ya que hacen parte de una nutrición balanceada y adecuados estilos de vida. Los hábitos alimenticios, la constante actividad física ayudan a mantener un peso adecuado y mejorar el rendimiento escolar, el bienestar emocional y cognitivo (Zegarra, Olivares, Solano, \& Musablon, 2011).

Por otra parte, Rojas et al. (2003) añade que los hábitos alimenticios son una serie de conductas y actitudes que tiene la persona al momento de alimentarse y que se adquieren durante la infancia. También son el resultado del comportamiento de las personas en el momento de preparar y consumir como una parte de sus costumbres sociales, culturales y religiosas, y que está influenciado por múltiples factores como los socioeconómicos, culturales y geográficos (Serafin, 2012).

Seguidamente la actividad física es otro estilo de vida de suma importancia en la edad escolar, según la OMS (2015) definiendo que la actividad física es un factor determinante del gasto de energía y reduce el riesgo relacionado con enfermedades no transmisibles, en especial las asociadas con la obesidad infantil. Asimismo; Tafur, Diaz, y Mauricio (2015) agregan que además de ser un movimiento corporal producido por los músculos esqueléticos, produce un gasto energético por encima de la tasa del metabolismo basal.

En las últimas décadas, la población peruana ha adoptado hábitos alimentarios poco saludables, como el consumo de comida rápida y barata con alto contenido calórico, y paralelamente ha disminuido la actividad física, probablemente por la estabilidad de la economía peruana, lo cual se refleja en la mayor capacidad adquisitiva en los hogares, también se ha notado cambios socioeconómicos en la población, sobre todo en las zonas urbanas como el transporte masivo que utiliza la población para desplazarse aún para tramos cortos, el uso de ascensores, el uso de la tecnología en la diversión que contribuye al incremento del sedentarismo, principalmente en los adultos y niños, estos últimos ven televisión en promedio 0,88 a 1,75 horas por día en programas comerciales (programas de entretenimiento) y 0,45 a 0,74 horas por día 13,14 programas no comerciales (programas y videos educativos) lo menciona (TarquiMamani, 2013).

Asimismo los medios de comunicación tienen gran influencia en las orientaciones hacia el consumo y en los comportamientos de la población escolar, por lo que sus mensajes pueden ser potenciadores o perjudiciales para la salud (Román y Quintana, 2010). Por otra parte, la evaluación nutricional que realizó el Ministerio de Salud (MINSA) a un millón 400 mil escolares beneficiarios del programa Qaliwarma durante el 2014, arrojó que el 23\% de ellos tiene problemas de sobrepeso y obesidad, mientras que un $12 \%$ sufre de anemia. La integrante del equipo técnico J.M. Oblitas (Plan de Salud Escolar, del 15 de marzo del 2014) explicó que el tamizaje del estado nutricional de este grupo de estudiantes escolares de zonas urbanas, se hizo mediante la medición de su peso y talla. Manifiesta: "Sabíamos que había problemas de anemia en los niños y niñas menores de cinco años, pero después de esa edad no existían estudios. Ahora tenemos evidencia de que efectivamente hay problemas de hábitos alimenticios en los escolares”.

Según el Ministerio de Salud, añade que los niños, deben alimentarse sanamente y hacerlo en las raciones correctas, ya que es un problema social peruano que afecta a muchísimos niños, obviamente más afectados se encuentran los niños en zonas rurales del Perú debido a la falta de recursos. En el sector rural se observa un porcentaje más alto (46\%) de hogares en donde los niños consumen menos proteínas diarias, mientras que en el sector urbano es de $22 \%$. Alrededor del $30 \%$ de niños a nivel nacional poseen un déficit calórico y también se observa que en las zonas con mayor pobreza el porcentaje de niños con déficit calórico está alrededor del 80\% mientras que en zonas de instituciones escolares privadas los niños con déficit calórico esta alrededor del 20\% (MINSA, 2015).

Además según los datos estadísticos, el departamento con mayor porcentaje de niños con desnutrición crónica es Huancavelica (44,7\%), Cajamarca (32,0\%), 
Huánuco (31,0\%), Apurímac (30,9\%), Ayacucho (30,3\%) y Cusco (29,1\%) siguiéndole Moquegua, el Callao y Lima. Estos niños por sus malos hábitos alimenticios no pueden prestar atención en las clases y hasta faltan por ello, entonces por más de que se trate de enseñar a estos niños, ellos no van a aprender si no pueden concentrarse. Por ello no solo debemos preocuparnos por la educación de ellos, sino también por la buena alimentación (MINSA, 2015)

Sin embargo las instituciones educativas públicas de este estudio no escapan de la realidad, no obstante el Ministerio de Salud (MINSA) viene desarrollando programas preventivos a lo largo de los años, cuya única finalidad es contribuir con la mejora de salud de los pobladores peruanos, especialmente en las etapas más vulnerables (niñez y adultez). En el pasado los niños tenían una mejor calidad de vida, ya que existía pocos distractores como la televisión, videojuegos, celulares, etc. Y se dedicaban mayormente a realizar las actividades cotidianas la cual contribuía al aprendizaje de hábitos saludables. En la actualidad, el impacto de la alimentación y la actividad física son los temas más resaltantes, debido a la gran influencia sobre el consumo de comida rápida y el sedentarismo por uso de la tecnología, como ver televisión por largas horas o jugar videojuegos y el uso de medios de transporte.

El Modelo teórico de enfermería utilizado en este estudio fue el de promoción de la salud de Nola Pender, (1996), enfocando en el estilo de vida (conductas alimenticias y actividad física). Es por ello que la infancia se considera un periodo crítico en la adquisición de hábitos alimentarios y de actividad física que serán perdurables en la edad adulta, como pilar básico en la prevención de las enfermedades no transmisibles principalmente el sobrepeso y la obesidad en la infancia (Correa y Gutiérrez, 2013). Estas consideraciones llevaron a realizar la presente investigación con el objetivo de determinar si existen diferencias en los hábitos alimentarios y actividad física en los escolares de cuarto a sexto grado del nivel primario de dos instituciones educativas públicas de Lima Este, 2015.

\section{MATERIALES Y MÉTODOS}

El estudio se realizó, en dos instituciones educativas públicas "5 de abril" ubicada en el distrito de Ate Vitarte- Huaycán donde los escolares vienen de familias de nivel socioeconómico bajo, obreros de manufactura, minas y construcción, o vendedores ambulantes, la mayoría de sus padres trabajan en Lima durante el día, los niños y los ancianos se encuentran generalmente abandonados, expuestos a numerosos riesgos. Por otro lado la institución educativa "María Goretti” ubicada en Ñaña - Lurigancho Chosica donde la mayoría de los escolares vienen de familias de nivel socioeconómico moderado. La investigación fue de enfoque cuantitativo, diseño no experimental, de corte transversal, tipo descriptivo - comparativo. El muestreo fue no probabilístico, tipo censal, dónde se incluyó a toda la población, destacando 311 escolares de la Institución "5 de Abril” y 260 escolares de la Institución "Santa María Goretti”, haciendo un total de 571 escolares, entre las edades de 9 a 12 años.

Los instrumentos usados fueron dos: los hábitos alimentarios se midieron con el test rápido KrecePlus, según la puntuación del test clasificados como bajo, medio o alto, con un Alfa de Crombach 0.87. La actividad física fue valorada mediante el test corto de actividad física Krecreplus, este test clasifica el estilo de vida basándose en la media de horas que ven la televisión o juegan con videojuegos y las horas de deporte extraescolar que realizan por semana, según la puntuación obtenida en el test, el nivel de actividad física del niño se clasifica en malo, regular y bueno, con un Alfa de Crombach 0.66. Estos instrumentos fueron validados Serra y Cols, (2003) en España. Debido que estos test eran validados por un País extranjero, en este estudio se realizó la validación por juicios de expertos de los instrumentos de hábitos alimentarios y actividad física a través de la prueba $\mathrm{V}$ de Aiken, lo cual evidenció una validez de contenido aceptable ( $\mathrm{V}>0.7)$.

La recolección de datos fue con previa autorización de los directores de las dos instituciones educativas públicas, y el consentimiento informado de los padres de los escolares. La información fue procesada con el paquete estadístico SPSS 22.0. Los resultados de la investigación se presentan en tablas simples y comparativas. Para verificar las hipótesis planteadas, se empleó la prueba de U de Mann Whitney y la Prueba $\mathrm{H}$ de Kruskal-Wallis, con el fín de determinar si existen o no diferencias significativas entre ambas instituciones, según género y lugar de procedencia. 
Hábitos alimenticios y actividad física en los escolares de cuarto a sexto grado de nivel primaria de dos instituciones educativas públicas de Lima Este, 2015

\section{RESULTADOS}

Tabla 1

Hábitos alimentarios y actividad física en los escolares de cuarto a sexto grado de nivel primaria de las dos instituciones educativas públicas de Lima Este.

\begin{tabular}{ccccccc}
\hline Colegio & Hábitos alimentarios & N & $\%$ & Actividad física & N & $\%$ \\
\hline 5 de Abril & Bajo & 9 & 2,9 & Malo & 159 & 51,1 \\
& Medio & 83 & 26,7 & Regular & 140 & 45,0 \\
& Alto & 219 & 70,4 & Bueno & 12 & 3,9 \\
& Total & 311 & 100,0 & Total & 311 & 100,0 \\
María Goretti & Bajo & 5 & 1,9 & Malo & 69 & 26,5 \\
& Medio & 53 & 20,4 & Regular & 178 & 68,5 \\
& Alto & 202 & 77,7 & Bueno & 13 & 5,0 \\
& Total & 260 & 100,0 & Total & 260 & 100,0 \\
\hline
\end{tabular}

En la tabla 1 se muestran los resultados de Hábitos alimentarios en los escolares de las dos instituciones educativas públicas de Lima Este. Destaca que los escolares de la Institución Educativa "5 de abril”, el 70.4\% tienen hábitos alimentarios alto y actividad física mala el 51,1\%. Por otro lado los escolares de la Institución Educativa "María Goretti”, el 77.7\% tienen hábitos alimentarios alto y actividad física regular 68,5\%.

Tabla 2

Hábitos alimentarios en los escolares de cuarto a sexto grado de nivel primaria de las dos instituciones educativas públicas de Lima Este según género.

\begin{tabular}{|c|c|c|c|c|c|c|c|c|}
\hline \multirow{3}{*}{$\begin{array}{c}\text { Institución } \\
\text { Género }\end{array}$} & \multicolumn{8}{|c|}{5 de Abril } \\
\hline & \multicolumn{2}{|c|}{ Bajo } & \multicolumn{2}{|c|}{ Medio } & \multicolumn{2}{|c|}{ Alto } & \multicolumn{2}{|c|}{ Total } \\
\hline & $\mathrm{N}$ & $\%$ & $\mathrm{~N}$ & $\%$ & $\mathrm{~N}$ & $\%$ & $\mathrm{~N}$ & $\%$ \\
\hline Masculino & 4 & $2,6 \%$ & 40 & $26,0 \%$ & 110 & $71,4 \%$ & 154 & $100,0 \%$ \\
\hline Femenino & 5 & $3,2 \%$ & 43 & $27,4 \%$ & 109 & $69,4 \%$ & 157 & $100,0 \%$ \\
\hline Total & 9 & $2,9 \%$ & 83 & $26,7 \%$ & 219 & $70,4 \%$ & 311 & $100,0 \%$ \\
\hline Institución & & & & & ría Go & & & \\
\hline \multirow[t]{2}{*}{ Género } & \multicolumn{2}{|c|}{ Bajo } & \multicolumn{2}{|c|}{ Medio } & \multicolumn{2}{|c|}{ Alto } & \multicolumn{2}{|c|}{ Total } \\
\hline & $\mathrm{N}$ & $\%$ & $\mathrm{~N}$ & $\%$ & $\mathrm{~N}$ & $\%$ & $\mathrm{~N}$ & $\%$ \\
\hline Masculino & 2 & $1,7 \%$ & 21 & $17,9 \%$ & 94 & $80,3 \%$ & 117 & $100,0 \%$ \\
\hline Femenino & 3 & $2,1 \%$ & 32 & $22,4 \%$ & 108 & $75,5 \%$ & 143 & $100,0 \%$ \\
\hline Total & 5 & $1,9 \%$ & 53 & $20,4 \%$ & 202 & $77,7 \%$ & 260 & $100,0 \%$ \\
\hline
\end{tabular}

En la tabla 2, se observa que de todos los escolares del género masculino de la institución "5 de abril”, el $71.4 \%$ de ellos tienen hábitos alimentarios altos, los escolares de género femenino el $69.4 \%$ tienen hábitos alimentarios altos. Por otro lado de todos los escolares de género masculino de la Institución
Educativa Pública “María Goretti”, el 80.3\% tienen hábitos alimentarios altos, y los escolares de género femenino, el 75.5\% tienen hábitos alimentarios altos. 
Tabla 3

Actividad Física en los escolares de cuarto a sexto grado de nivel primaria de las dos instituciones educativas públicas de Lima Este según género.

\begin{tabular}{|c|c|c|c|c|c|c|c|c|}
\hline \multirow{3}{*}{$\begin{array}{c}\text { Institución } \\
\text { Género }\end{array}$} & \multicolumn{8}{|c|}{5 de Abril } \\
\hline & \multicolumn{2}{|c|}{ Malo } & \multicolumn{2}{|c|}{ Regular } & \multicolumn{2}{|c|}{ Bueno } & \multicolumn{2}{|c|}{ Total } \\
\hline & N & $\%$ & N & $\%$ & N & $\%$ & $N$ & $\%$ \\
\hline Masculino & 80 & $51,9 \%$ & 70 & $45,5 \%$ & 4 & $2,6 \%$ & 154 & $100,0 \%$ \\
\hline Femenino & 79 & $50,3 \%$ & 70 & $44,6 \%$ & 8 & $5,1 \%$ & 157 & $100,0 \%$ \\
\hline Total & 159 & $51,1 \%$ & 140 & $45,0 \%$ & 12 & $3,9 \%$ & 311 & $100,0 \%$ \\
\hline Institución & \multicolumn{8}{|c|}{ María Goretti } \\
\hline \multirow[t]{2}{*}{ Género } & \multicolumn{2}{|c|}{ Malo } & \multicolumn{2}{|c|}{ Regular } & \multicolumn{2}{|c|}{ Bueno } & \multicolumn{2}{|c|}{ Total } \\
\hline & $\mathrm{N}$ & $\%$ & $\mathrm{~N}$ & $\%$ & $\mathrm{~N}$ & $\%$ & $\mathrm{~N}$ & $\%$ \\
\hline Masculino & 33 & $28,2 \%$ & 77 & $65,8 \%$ & 7 & $6,0 \%$ & 117 & $100,0 \%$ \\
\hline Femenino & 36 & $25,2 \%$ & 101 & $70,6 \%$ & 6 & $4,2 \%$ & 143 & $100,0 \%$ \\
\hline Total & 69 & $26,5 \%$ & 178 & $68,5 \%$ & 13 & $5,0 \%$ & 260 & $100,0 \%$ \\
\hline
\end{tabular}

En la tabla 3 se observa que de todos los escolares del género masculino de la institución "5 de abril”, el 51.9\% de ellos tienen actividad física mala y los escolares de género femenino el 50.3\% tienen actividad física mala. Por otro lado de todos los escolares de género masculino de la Institución
Educativa Pública “María Goretti”, el 65.8\% tienen actividad física regular y los escolares de género femenino, el 70.6\% tienen actividad física regular.

Tabla 4

Comparación de hábitos alimentarios y actividad física en los escolares de cuarto a sexto grado de nivel primaria de las dos instituciones públicas de Lima Este según género a través de la prueba de la U de Mann Whitney.

\begin{tabular}{|c|c|c|c|c|c|c|c|}
\hline & & & Hábitos alimentar & & & & \\
\hline Instituciones & Genero & N & Rango promedio & $\begin{array}{l}\text { Suma de } \\
\text { rangos }\end{array}$ & $\begin{array}{l}\text { U de Mann- } \\
\text { Whitney }\end{array}$ & Z & $P$ \\
\hline 5 de Abril & Masculino & 154 & 161,56 & 24880,50 & 11232.5 & -1.092 & 0.275 \\
\hline & Femenino & 157 & 150,54 & 23635,50 & & & \\
\hline & Total & 311 & & & & & \\
\hline María Goretti & Masculino & 117 & 134,70 & 15759,50 & 7874.5 & -0.821 & 0.412 \\
\hline & Femenino & 143 & 127,07 & 18170,50 & & & \\
\hline & Total & 260 & & & & & \\
\hline & & & Actividad física & & & & \\
\hline Instituciones & Género & N & Rango promedio & $\begin{array}{l}\text { Suma de } \\
\text { rangos }\end{array}$ & $\begin{array}{l}\text { U de Mann- } \\
\text { Whitney }\end{array}$ & Z & $p$ \\
\hline 5 de Abril & Masculino & 154 & 154,50 & 23793,00 & $11,858,000$ &,- 295 & ,768 \\
\hline & Femenino & 157 & 157,47 & 24723,00 & & & \\
\hline & Total & 311 & & & & & \\
\hline María Goretti & Masculino & 117 & 131,55 & 15391,50 & $8,242,500$ &,- 208 & ,835 \\
\hline & Femenino & 143 & 129,64 & 18538,50 & & & \\
\hline & Total & 260 & & & & & \\
\hline
\end{tabular}

En la tabla 4 se observa que en los hábitos alimentarios y actividad física de los escolares según género no hay diferencias estadísticamente significativas entre los puntajes alcanzados $(p>, 05)$ en ambas instituciones educativas públicas. 
Hábitos alimenticios y actividad física en los escolares de cuarto a sexto grado de nivel primaria de dos instituciones educativas públicas de Lima Este, 2015

Tabla 5

Hábitos alimentarios en los escolares de cuarto a sexto grado de nivel primario de las dos instituciones educativas públicas de Lima Este según lugar de procedencia.

\begin{tabular}{|c|c|c|c|c|c|c|c|c|}
\hline \multirow{3}{*}{$\frac{\text { Institución }}{\text { Lugar de procedencia }}$} & \multicolumn{8}{|c|}{5 de Abril } \\
\hline & \multicolumn{2}{|c|}{ Bajo } & \multicolumn{2}{|c|}{ Medio } & \multicolumn{2}{|c|}{ Alto } & \multicolumn{2}{|c|}{ Total } \\
\hline & $\mathrm{N}$ & $\%$ & $\mathrm{~N}$ & $\%$ & $\mathrm{~N}$ & $\%$ & $n$ & $\%$ \\
\hline Costa & 6 & $3,2 \%$ & 49 & $26,3 \%$ & 131 & $70,4 \%$ & 186 & $100,0 \%$ \\
\hline Sierra & 2 & $2,8 \%$ & 19 & $26,4 \%$ & 51 & $70,8 \%$ & 72 & $100,0 \%$ \\
\hline Selva & 1 & $1,9 \%$ & 15 & $28,3 \%$ & 37 & $69,8 \%$ & 53 & $100,0 \%$ \\
\hline Total & 9 & $2,9 \%$ & 83 & $26,7 \%$ & 219 & $70,4 \%$ & 311 & $100,0 \%$ \\
\hline Institución & \multicolumn{8}{|c|}{ María Goretti } \\
\hline \multirow[t]{2}{*}{ Lugar de procedencia } & \multicolumn{2}{|c|}{ Bajo } & \multicolumn{2}{|c|}{ Medio } & \multicolumn{2}{|c|}{ Alto } & \multicolumn{2}{|c|}{ Total } \\
\hline & $\mathrm{N}$ & $\%$ & $\mathrm{~N}$ & $\%$ & $\mathrm{~N}$ & $\%$ & $\mathrm{~N}$ & $\%$ \\
\hline Costa & 2 & $2,5 \%$ & 10 & $12,3 \%$ & 69 & $85,2 \%$ & 81 & $100,0 \%$ \\
\hline Sierra & 0 & $0,0 \%$ & 20 & $19,2 \%$ & 84 & $80,8 \%$ & 104 & $100,0 \%$ \\
\hline Selva & 3 & $4,0 \%$ & 23 & $30,7 \%$ & 49 & $65,3 \%$ & 75 & $100,0 \%$ \\
\hline Total & 5 & $1,9 \%$ & 53 & $20,4 \%$ & 202 & $77,7 \%$ & 260 & $100,0 \%$ \\
\hline
\end{tabular}

En la tabla 5 se observa que la institución educativa 5 de abril en la costa, el $70.4 \%$ tienen hábitos alimentarios altos, sierra el 70.8\% tienen hábitos alimentarios altos, y la selva el 69.8\% tienen hábitos alimentarios altos. Por otro lado, de todos los escolares de la costa de la institución educativa María Goretti el 85.2\% tienen hábitos alimentarios altos, la sierra el $80.8 \%$ tienen hábitos alimentarios altos y la selva el 65.3\% tienen hábitos alimentarios altos.

Tabla 6

Actividad física en los escolares de cuarto a sexto grado de nivel primaria de las dos instituciones educativas públicas de Lima Este según lugar de procedencia.

\begin{tabular}{|c|c|c|c|c|c|c|c|c|}
\hline \multirow{3}{*}{$\begin{array}{c}\text { Institución } \\
\text { Lugar de procedencia }\end{array}$} & \multicolumn{8}{|c|}{5 de Abril } \\
\hline & \multicolumn{2}{|c|}{ Malo } & \multicolumn{2}{|c|}{ Regular } & \multicolumn{2}{|c|}{ Bueno } & \multicolumn{2}{|c|}{ Total } \\
\hline & $\mathrm{N}$ & $\%$ & $\mathrm{~N}$ & $\%$ & $\mathrm{~N}$ & $\%$ & N & $\%$ \\
\hline Costa & 90 & $48,4 \%$ & 88 & $47,3 \%$ & 8 & $4,3 \%$ & 186 & $100,0 \%$ \\
\hline Sierra & 43 & $59,7 \%$ & 27 & $37,5 \%$ & 2 & $2,8 \%$ & 72 & $100,0 \%$ \\
\hline Selva & 26 & $49,1 \%$ & 25 & $47,2 \%$ & 2 & $3,8 \%$ & 53 & $100,0 \%$ \\
\hline Total & 159 & $51,1 \%$ & 140 & $45,0 \%$ & 12 & $3,9 \%$ & 311 & $100,0 \%$ \\
\hline Institución & \multicolumn{8}{|c|}{ María Goretti } \\
\hline \multirow[t]{2}{*}{ Lugar de procedencia } & \multicolumn{2}{|c|}{ Malo } & \multicolumn{2}{|c|}{ Regular } & \multicolumn{2}{|c|}{ Bueno } & \multicolumn{2}{|c|}{ Total } \\
\hline & $\mathrm{N}$ & $\%$ & $\mathrm{~N}$ & $\%$ & $\mathrm{~N}$ & $\%$ & N & $\%$ \\
\hline Costa & 14 & $17,3 \%$ & 62 & $76,5 \%$ & 5 & $6,2 \%$ & 81 & $100,0 \%$ \\
\hline Sierra & 25 & $24,0 \%$ & 72 & $69,2 \%$ & 7 & $6,7 \%$ & 104 & $100,0 \%$ \\
\hline Selva & 30 & $40,0 \%$ & 44 & $58,7 \%$ & 1 & $1,3 \%$ & 75 & $100,0 \%$ \\
\hline Total & 69 & $26,5 \%$ & 178 & $68,5 \%$ & 13 & $5,0 \%$ & 260 & $100,0 \%$ \\
\hline
\end{tabular}

En la tabla 6, muestra que los escolares de la institución educativa "5 de abril” en la costa, el 48.4\% tienen actividad física mala, la sierra el 59.7\% tienen actividad física mala, y la selva el $49.1 \%$ tienen actividad física mala. Por otro lado, de todos los escolares de la institución educativa "María Goretti” en la costa el $76.5 \%$ tienen actividad física regular, la sierra el $69.2 \%$ tienen actividad física regular y la selva se observa que el $58.7 \%$ tienen actividad física regular. 
Tabla 7

Comparación de hábitos alimentarios y actividad física en los escolares de cuarto a sexto grado de nivel primaria, de dos instituciones educativas públicas Lima Este según lugar de procedencia a través de la prueba Prueba de Kruskal-Wallis.

\begin{tabular}{|c|c|c|c|c|c|c|}
\hline \multicolumn{7}{|c|}{ Hábitos alimentarios } \\
\hline Institución & Lugar de Procedencia & $\mathrm{N}$ & Rango promedio & Chi-cuadrado & gl & $P$ \\
\hline \multirow[t]{4}{*}{5 de Abril } & Costa & 186 & 160,48 & 1,288 & 2 &, 525 \\
\hline & Sierra & 72 & 151,63 & & & \\
\hline & Selva & 53 & 146,21 & & & \\
\hline & Total & 311 & & & & \\
\hline \multirow[t]{4}{*}{ María Goretti } & Costa & 81 & 146,98 & 10,071 & 2 & ,007 \\
\hline & Sierra & 104 & 132,89 & & & \\
\hline & Selva & 75 & 109,39 & & & \\
\hline & Total & 260 & & & & \\
\hline \multicolumn{7}{|c|}{ Actividad física } \\
\hline Institución & Lugar de procedencia & $\mathrm{N}$ & Rango promedio & Chi-cuadrado & gl & $p$ \\
\hline \multirow[t]{4}{*}{5 de Abril } & Costa & 186 & 163,34 & 5,570 & 2 & ,062 \\
\hline & Sierra & 72 & 134,51 & & & \\
\hline & Selva & 53 & 159,45 & & & \\
\hline & Total & 311 & & & & \\
\hline \multirow[t]{4}{*}{ María Goretti } & Costa & 81 & 148,30 & 16,520 & 2 &, 000 \\
\hline & Sierra & 104 & 136,95 & & & \\
\hline & Selva & 75 & 102,33 & & & \\
\hline & Total & 260 & & & & \\
\hline
\end{tabular}

Tabla 3 se observa que en los hábitos alimentarios de los escolares según el lugar de procedencia muestra que la institución "5 de Abril" no hay diferencias estadísticamente significativas entre los puntajes alcanzados $\left(\chi^{2}=5,570 ; \mathrm{p}>, 05\right)$. No obstante se encontró diferencia estadisticamente significativa en la institución educativa "María Goretti" $\left(\chi^{2}=16,520\right.$; $\mathrm{p}<5$ : ,000).
Por otro lado se observa que en la actividad física de los escolares de la institución educativa "5 de “Abril según lugar de procedencia no hay diferencias estadísticamente significativas entre los puntajes alcanzados $\left(\chi^{2}=5,570 ; \mathrm{p}>, 05\right)$. No obstante se encontró diferencia estadísticamente significativa en la institución educativa "María Goretti" $\left(\chi^{2}=16,520\right.$; $\mathrm{p}<5$ : ,000).

\section{Tabla 8}

Comparación de hábitos alimentarios en los escolares de cuarto a sexto grado de nivel primaria según el tipo de institución educativa pública de Lima este a través de la prueba de la U de Mann-Whitney.

\begin{tabular}{lllllll}
\hline Institución & $\mathrm{N}$ & $\begin{array}{l}\text { Rango } \\
\text { promedio }\end{array}$ & $\begin{array}{l}\text { Suma de } \\
\text { rangos }\end{array}$ & $\begin{array}{l}\text { U de Mann- } \\
\text { Whitney }\end{array}$ & Z & P \\
\hline 5 de Abril & 311 & 265,84 & 82675,00 & $34,159,000$ & $-3,222$ &, 001 \\
María Goretti & 260 & 310,12 & 80631,00 & & & \\
Total & 571 & & & & & \\
\hline
\end{tabular}

En la tabla 4 se observa que existen diferencias estadísticamente significativas entre los puntajes alcanzados $(\mathrm{Z}=-3,222 ; \quad \mathrm{p}<0.001)$ en ambas instituciones educativas públicas. 
Hábitos alimenticios y actividad física en los escolares de cuarto a sexto grado de nivel primaria de dos instituciones educativas públicas de Lima Este, 2015

Tabla 9

Comparación de la Actividad física en los escolares de cuarto a sexto grado de nivel primaria según el tipo de institución educativa pública de Lima Este a través de la prueba de la U de Mann-Whitney.

\begin{tabular}{lrlllcc}
\hline \multicolumn{1}{c}{ Institución } & N & Rango promedio & Suma de rangos & U de Mann-Whitney & Z & p \\
\hline 5 de Abril & 311 & 244,29 & 75973,50 & $27,457,500$ & $-6,707$ &, 000 \\
Maria Goretti & 260 & 335,89 & 87332,50 & & & \\
Total & 571 & & & & & \\
\hline
\end{tabular}

En la tabla 5 se observa que existe diferencias estadísticamente significativas entre los puntajes alcanzados $(Z=-6,707 ; \mathrm{p}<.000)$ en ambas instituciones educativas públicas.

\section{DISCUSIÓN}

Los hábitos alimenticios y la actividad física en escolares, son temas que deberían estar presentes en todas las programaciones que se planteen en la educación primaria, de manera que ayude a combatir los problemas relacionados con la alimentación y la actividad física dentro y fuera de la escuela, esto con el objetivo de mostrar la importancia de adquirir hábitos saludables, como una dieta equilibrada desde edades muy tempranas e inculcarles una actitud crítica ante un determinado tipo de alimentación y la publicidad de éstos.

El propósito del estudio fue determinar si existen diferencias significativas en los hábitos alimentarios y actividad física de los escolares de cuarto a sexto del nivel primario de dos instituciones públicas de Lima Este. En primer lugar se describe los hábitos alimentarios, mostrando que los escolares presentaron hábitos alimentarios altos y medios respectivamente. En la Institución "5 de abril”, el 70.4\% (219) de los escolares tienen hábitos alimentarios alto, y el 26,7\% (83) medio. Así mismo los escolares de la Institución “María Goretti” el 77.7\% (202) tienen hábitos alimentarios alto y el 20.4\% (53) medios. Estos resultados obtenidos, indican que los hábitos alimentarios de los escolares son adecuados, debido a que en ambas instituciones se desarrollan programas preventivos como "las escuelas saludables" la cual favorece a los escolares a tener un mejor estilo de vida. Sin embargo existe un porcentaje de escolares que tiene hábitos alimentarios medios, lo cual están influenciados por múltiples factores como los socioeconómicos, culturales y geográficos (Serafin, 2012).

Asimismo la sociedad ha obligado a los padres a trabajar fuera de casa, por lo que los niños se encuentran solos la mayor parte del día, y para su alimentación deben acudir al consumo de alimentos fuera de su casa, por ende seleccionando alimentos ricos en grasa y azúcar (Lewandowski, 2015). Un estudio realizado por Castañeda, Rocha, y Ramos, (2008) a 69 alumnos de una escuela mexicana, mostró que más de la mitad de la población estudiada presentaron hábitos alimentarios inadecuados, podría deberse por la ingesta de alimentos rápidos o la llamada "comida chatarra”, o la falta de un programa educativo en salud, sumado a la inactividad física de los preadolescentes. Concluyeron que los hábitos alimentarios deficientes, pueden conllevar al escolar a presentar sobrepeso u obesidad en el futuro.

Los hábitos alimentarios nacen en la familia, se desarrollan normalmente durante la infancia y en particular, durante la adolescencia, se contrastan en la comunidad en contacto con los padres y con el medio social, además de eso es notable que existen factores que conllevan a que los escolares tengan este tipo de hábitos alimentarios, entre ellos está el no desayunar antes de ir a clase, ingerir golosinas, o incluir alimentos que no son beneficiosos para la salud. El estudio mostró que el 24.4\% (76) de los escolares del colegio " 5 de abril" no toman desayuno, a diferencia de los estudiantes del colegio "María Goretti" en un 10.0\%(26) (Ver Apéndice 5)

En el caso del niño que va al colegio de mañana, hay que asegurarse de que se levante con el tiempo suficiente para desayunar. Esas horas sin haber desayunado serán muy intensas, a pesar de que pasará la mayor parte del tiempo sentado. La actividad física no es la única que requiere energías. La actividad mental también necesita un buen aporte (Tafur, et al.2015).

Sin embargo el estudio de Correa y Gutiérrez, (2013) en escolares de 11 y 12 años en la provincia de Granada, muestra resultados diferentes a través del test Krece plus el 85,1\% si desayunan antes de ir al colegio, pero la composición del desayuno es inadecuada. Por lo tanto, esto es un indicador de que la gran mayoría de los escolares no toma un desayuno correcto basado en el consumo de leche o derivados, cereales o pan y fruta o zumo de fruta. Esta tarea es 
una gran responsabilidad de los padres, ya que son los que deben velar por la alimentación de sus hijos. Sin embargo, los tutores y profesores también cumplen un papel importante en el proceso. La infancia es un periodo decisivo en la vida de un niño, por lo que su alimentación debe ser equilibrada desde el primer momento (Bayona, 2013).

Además de eso en el ítem desayunas un lácteo (Ver apéndice 5) se encontró que en ambas instituciones educativas el 37,5\% (107) de los escolares no desayunaban un lácteo, esto puede deberse a que la mayoría prefiere tomar refresco o té. Para muchas personas, el consumo de lácteos (principal fuente de calcio) está asociado con el desayuno, de manera que si éste no se realiza, podrían producirse deficiencias de este nutriente que repercutiría en la salud ocasionando, alteraciones del crecimiento, desarrollo y osteoporosis en la etapa adulta (Carbajal y Pinto, 2015). Un estudio similar por Correa y Gutiérrez (2013) en escolares con edades comprendidas entre 11 y 12 años de edad en la provincia de Granada, evidencia que el $25,5 \%$ de los escolares no tomaba un lácteo en el desayuno, por diversas situaciones como en salir tarde a clases, a otros simplemente no les gusta o prefieren tomar café. EROSKI, (2015) añade que la leche en sí constituye el mejor aporte de calcio, proteínas y nutrientes necesarios para la formación de huesos y dientes. En la infancia y la adolescencia aporta la energía y las vitaminas A y D, en la edad adulta favorece la conservación de la masa ósea, por lo tanto es de suma importancia en la etapa escolar, ya que una buena alimentación dará buenos resultados en la salud y en el rendimiento académico.

Aparte de ello, cabe destacar que el consumo de frutas en el desayuno, forma parte de un hábito alimentario positivo. El estudio muestra que en ambas instituciones el 64,1\% (186) no toman una fruta o jugo de fruta todos los días, esto puede ser porque en la escuela hay kioscos que ofrecen alimentos etiquetados como comida chatarra, y poca estimulación al consumo de frutas y verduras. Si bien Llargués et al. (2009) dietary patterns and exercise habits in children attending the first year of primary school in the city of Granollers Spain realizaron otro estudio similar en escolares de 6 escuelas públicas de Barcelona, pero obtuvieron resultados con menor porcentaje el 29\% de los escolares no consumían una fruta durante el día. Una vez más, los resultados del presente estudio aunque son menores enfatizan la importancia de los hábitos alimentarios de los niños.

Respecto al consumo de hamburguesas, hot dog, pizza, se encontró un $73.6 \%$ (214) y el 61.2\% (181) de los escolares consumen golosinas o dulces en ambas instituciones educativas, puesto que las etiquetas de estos "alimentos" son llamativas para los niños. Si bien es cierto este no es el único causante, sino la publicidad de estos. En cuanto a lo anterior mencionado, la televisión, ha contribuido a la modificación de los hábitos alimentarios de los niños debido a que representan un grupo de edad crítico y fácil de manipular en cuanto al consumo de nuevos alimentos, que la mayor parte de las veces no son saludables (Macias, et al.2012).

En la misma línea se menciona que los medios de comunicación tienen gran influencia en las orientaciones hacia el consumo y en los comportamientos de la población escolar, por lo que sus mensajes pueden ser potenciadores o perjudiciales para la salud (Román y Quintana, 2010). La encuesta Nacional de Salud (2006) en España, indica que el $22,13 \%$ de los escolares entre 5 y 9 años y el $16,71 \%$ entre 10 y 15 años, ven 3 horas diarias de TV. Diversos estudios relacionan obesidad en niños con estas actividades, no solo por ser actividades sedentarias, sino que muchos de ellos comen comidas muy energéticas mientras ven la TV.

En la población estadounidense se estimó que los niños destinan 21 a 22 horas semanales a ver televisión; de las cuales, alrededor de 3 horas correspondieron a publicidad de alimentos. En un 91\% de los casos, esta publicidad se refiere a alimentos con un elevado contenido de grasa, azúcar o sal. Adicionalmente, estudios en niños de cuarto a quinto grado han observado que independientemente de su sexo, capacidad de lectura, factores étnicos, ocupación de los padres y nivel educativo de éstos, el consumo de televisión correlacionaba positivamente con conceptos erróneos sobre los alimentos, nutrición y los malos hábitos alimentarios. Se ha indicado además que, existe una correlación entre la elección de alimentos de niños de 3 a 8 años y la publicidad de estos alimentos por televisión (Olivares y cols., 2003).

Es por ello, en el ámbito familiar se detectan malos hábitos alimentarios, lo que seguramente condiciona la falta de adquisición o seguimiento de mejores hábitos del escolar. Sin embargo hay padres muy permisivos, o simplemente que no están conectados con sus hijos para enseñarles la práctica de buenos hábitos alimenticios, porque estos hábitos no solo se adquieren en el hogar sino también en la escuela, no obstante en los kioscos escolares donde se encuentran “alimentos” con alto contenido energético, de grasas y de sodio, por ejemplo, papas fritas, milanesas, hamburguesas, sándwiches, frituras, alfajores, gaseosas, chocolates, y otras golosinas. A pesar de que dichos "alimentos" son de baja calidad 

públicas de Lima Este, 2015

nutricional, siguen siendo los preferidos por los niños, ya que están sustentados por una enorme carga publicitaria (Funes, 2010). Es aquí donde los padres deben intervenir en las costumbres de los hijos; pero también es responsabilidad de los educadores, por lo que desde la escuela debemos intervenir para promover buenos hábitos en los alumnos (Bayona, 2013).

En cuanto a los hábitos alimentarios según género, los resultados puntúan dentro de los rangos altos y medio en ambos géneros. Siendo que los escolares de la Institución “5 de abril”, el género masculino 71.4\% (110) de ellos tienen hábitos alimentarios altos y el 26.0\% (40) presentan hábitos medio, seguidamente en el género femenino el 69.4\% (109) tienen hábitos alimentarios altos y el 27.4\% (43) medio. Por otro lado, los escolares de la Institución "María Goretti" muestra que del género masculino, el 80.3\% (94) tienen hábitos alimentarios altos y el 17.9\% (21)son medios, mientras que el género femenino el 75.5\% (108) tienen hábitos alimentarios altos y el 22.4\% (32) son medios. Sin embargo se observa que no hay diferencias estadísticamente significativas del género en ambas instituciones, entre los puntajes alcanzados $(Z=-1,092 ;-8: p>, 05)$.

Espinosa (2015) desarrolló un estudio semejante donde uno de sus objetivos fue describir los hábitos alimentarios por género, en escolares de 5 a 12 años en Ecuador, donde evidenciaron que el 85,7\% presentaban hábitos alimentarios no adecuados (medios y bajos), en hombres representado por el $91.4 \%$ y en las mujeres el $78 \%$. En concordancia con lo anterior Orgiles et al., (2014) mostró con respecto a los hábitos de alimentación de los niños varones 10 a 12 años en la provincia de Alicante - España, según su categoría ponderal, sin embargo en este estudio si se han encontrado diferencias estadísticamente significativas en un $34 \%$ de los cuales presentan hábitos alimentarios medios y bajos, debido a un factor importante que influye en la alimentación, es decir el lugar donde comen de forma habitual, así como el hábito de no tomar desayuno y poco almuerzo. En las niñas sólo se encontraron diferencias estadísticamente significativas del $24 \%$ que consistía en tomar doble cena.

Los resultados que se muestran en el estudio anterior, sugieren que los niños que comen en lugares no apropiados o menos comidas al día, pueden estar indicando, como el estudio de Toschke, Helmut Ku y Berthold Koletzko (2005) un factor de riesgo para la obesidad y el sobrepeso infantil como consecuencia de estos malos hábitos alimentarios. En relación a los estudios mencionados, en este estudio se ha encontrado que las mujeres presentan con mayor relevancia hábitos alimentarios inadecuados (medios) por lo que puede ser que tenga mucha influencia estos factores, de no comer a la hora indicada o en los kioscos del colegio, donde la mayoría de los alimentos no son adecuados para el consumo humano, especialmente para un escolar que está en la etapa de desarrollo.

Respecto a los hábitos alimentarios según el lugar de procedencia de los escolares se encontró que en la institución “5 de abril” tienen hábitos alimentarios altos, en la región sierra el 70.8\%(51), el 70.4\%(131) costa y el 69.8\%(37) selva, así mismo en los hábitos inadecuados (medios) el 28.3\%(15)de la selva, el $26.4 \%$ (19) sierra y el 26.3\% (49) costa. Por otra lado en la Institución “María Goretti” los escolares que tienen hábitos alimentarios altos, en la costa 85.2\%(69), sierra 80.8\%(84) y 65.3\%(49) selva, de igual manera en los hábitos alimentarios inadecuados (medios), el 46,7\%(35) son de la selva, el 43,2\% (35) costa y el $41,3 \%$ de la sierra, sin embargo no se observa diferencias estadísticas significativas $\left(\chi^{2}=1,288 ; p>, 05\right)$ en ambas instituciones.

Cabe resaltar que la mayoría de resultados predominan los rangos altos y medios en las tres regiones, obteniendo menor porcentaje la región selva, esto se debe a que los escolares sufren un proceso de adaptación, especialmente en la alimentación, ya que tiene mucha influencia la cultura. En casi todos los países, los factores sociales y culturales tienen una influencia muy grande sobre lo que come la gente, como preparan sus alimentos, las prácticas alimentarias y los alimentos que prefieren. Es verdad que algunas prácticas alimentarias tradicionales y tabúes de ciertas sociedades pueden contribuir a deficiencias nutricionales en grupos específicos de la población, por lo tanto tener un buen estado de salud depende de una buena nutrición la que se obtiene de una alimentación equilibrada, una buena calidad de producción de los alimentos, buenos hábitos alimentarios y el desarrollo alcanzado por una comunidad (Guerrero, 2005).

Asimismo si descubrimos dónde, cuándo, en compañía de quién se consumen los alimentos y el conjunto de las relaciones sociales que prevalecen en esa sociedad, los hábitos alimentarios constituyen una parte integrada de la totalidad cultural, la comida proporciona importantes aspectos de identidad sociocultural, lo cual expresa la forma en que los individuos de diferentes sociedades proyectan sus identidades. Espino, Valladares, y Satalaya, (2004) desarrollaron un estudio en Perú, donde uno de sus objetivos fue identificar los factores culturales 
que intervienen en el proceso de alimentación de la población infantil en Ashaninka y Aguaruna, se evidenció que uno de los factores principales es la región, donde vives o de donde naciste, afirma que tiene mucha influencia en los hábitos alimentarios, ya que involucra las costumbres, las conductas de acuerdo a la región ya sea costa, sierra o selva. De igual manera en este estudio de investigación predominaron los hábitos inadecuado (medio) en la región selva, posiblemente también se deba a los factores culturales de cada familia la cual integra cada uno de estos escolares.

Así mismo datos interesantes se encontraron en un estudio desarrollado por Ferrer y Yépez citado por Calle y Montaleza (2011) sobre “Obesidad y Salud” a 1800 niños de varias escuelas particulares, fiscales, mixtas, matutinas y vespertinas donde muestra que en la Costa es el $16 \%$ y en la Sierra el $11 \%$ de los menores, tienen tendencia a la obesidad. En las ciudades principales del país: en Guayaquil la prevalencia es del 16\%y en Quito es del 9.6\%. Las niñas registran los índices más altos con el 15\%, frente a los niños que llegan al 12\% quienes concluyen que los niños de la costa tienen más probabilidad de sufrir obesidad o sobrepeso como una consecuencia de los malos hábitos alimentarios que estos tienen. A diferencia de los resultados de este estudio, el porcentaje de escolares con hábitos alimentarios no adecuados es la selva, lo que es un indicativo relevante de que no se está tomando las medidas apropiadas para evitar que los escolares enfermen o lleguen a tener obesidad o sobrepeso como lo menciona en el estudio anterior no hay un seguimiento de los programas de salud en la escuela.

Respecto a la actividad física que es fundamental en todas las edades, desempeña un papel importante en la prevención de enfermedades no transmisibles ya que está vinculada estrechamente con los estilos de vida saludable. En la etapa escolar, si bien se realiza actividad física como parte de un curso, sin embargo es de suma importancia hacer actividad física extraescolar. Actualmente se tiene una gran conciencia sobre los beneficios que aporta la práctica regular de actividad física, que junto con buenos hábitos alimentarios, puede ayudar a evitar o retardar la manifestación de ciertas enfermedades. En este sentido los hábitos alimentarios y la actividad física en la niñez permitirán actuar en consecuencia, definir los estilos y la calidad de vida en la etapa adulta.

Referente a ello, al describir la actividad física de los escolares de cuarto a sexto grado de las dos instituciones se encontró que en ambas instituciones educativas públicas, la mayoría de los escolares puntúan dentro de los rangos de actividad física regular y mala. En la Institución "5 de Abril” el $51.1 \%$ (159) es mala, y solo el 45.0\% (140) regular. De igual manera en la Institución "María Goretti”, el 68.5\% (178) es regular, y sólo el 26.5\% (69) mala. Es por ello, Peña y Bacallao (2001) añaden a la inactividad física como problema de salud pública es relativamente reciente, puesto que el desarrollo económico y social de los pueblos ha llevado a estilos de vida cada vez más sedentarios, asimismo el desarrollo tecnológico, los niños se tornan más sedentarios, aumentando la prevalencia de sobrepeso y obesidad en todo el mundo. Así mismo Correa y Gutiérrez (2013) realizaron un estudio similar, en escolares de 8 a 12 años, en Ecuador, donde el 53.2\% de la población escolar tenía un nivel de actividad física regular o malo y un 66\% invertía 2 o más horas al día en actividades sedentarias.

Esto se debe tanto al ejercicio como al sedentarismo, una vida sedentaria, vinculada al cambio en los hábitos de ocio, con un incremento del número de horas que dedican a ver la televisión influye en la vida de los escolares, donde presentan un signo de alarma. Por lo tanto, concluyen que mediante programas de intervención educativa se debe promover el ejercicio físico, incidiendo en la importancia de reducir las actividades sedentarias.

Añadiendo al estudio anterior, Martínez et al. (2010) realizaron un estudio donde uno de sus objetivos fue describir la actividad física de los niños de 6-10 años de Barcelona, evidencian que el 35,9\% de la población estudiada no practicaba ninguna actividad deportiva extraescolar, y que un 51,8\% practicaba menos de 2 horas a la semana. Por otra parte, hasta un 63,4\% invierte 2 o más horas al día en actividades sedentarias como ver la televisión o jugar con videojuegos. De acuerdo con estos datos, se consideró que un 58,7\% de la población presentó un mal estilo de vida y que sólo un 3,6\% tiene bueno.

Además de ello, en este estudio se encontró que uno de los ítems, cuántas horas dedicas a ver televisión o juegas videojuegos de los escolares, en la Institución "5 de abril", muestra que los escolares ven 4 horas con un 24.1\% (75), y el 23.8\% (74) ven 2 horas. Seguidamente la Institución “María Goretti” muestra que los escolares ven 4 horas al 39.6\% (103) y el 28.5\% (74) ve 3 horas de televisión, es decir los escolares pasan varias horas viendo televisión o videojuegos, por eso es importante que el escolar tenga un cronograma de actividades para cada día, organizado de tal forma que contribuya a tener un mejor estilo de vida. Por ello, es conveniente reducir el tiempo que dedicamos a tareas o actividades sedentarias como sentarnos frente al televisor o jugar videojuegos por varias horas 

públicas de Lima Este, 2015

(Correa , Gutiérrez, 2013). Así mismo se realizó un estudio en escolares de 11-12 años en la provincia de Granada, a través del test corto Krece Plus y mostró que el 66\% de los niños invierte 2 o más horas al día en actividades sedentarias como ver la televisión o jugar con videojuegos. Según Alustiza, citada por De los Angeles y Rodriguez (2009) afirma que ver la TV es la actividad que nuestros niños dedican más tiempo, lo que reduce la actividad física y promueve la ingesta excesiva de alimentos promocionados por la propia $\mathrm{TV}$, generando actitudes y representaciones negativas.

Según Arboccó y Arboccó (2012) afirma que la Televisión presenta estímulos audiovisuales, los cuales son más efectivos que los visuales y auditivos por sí solos. Se impone sobre los otros medios de comunicación por penetrar en el hogar, en la vida diaria y llegar a formar parte del cúmulo de hábitos de cualquier persona de nuestra época. Muchos padres permiten a sus hijos ver solos la TV, sin limitar el horario ni seleccionar la programación, por ello las principales medidas preventivas que recomienda: No comer mientras se ve la TV, disminuir las horas de TV, evitar la TV en los cuartos de los niños (Barbancho Cisneros, 2005). De igual manera Doak et al. (2006) afirma que la educación física en las escuelas y la reducción de las horas que ven la televisión son dos ejemplos de intervenciones que han tenido éxito para reducir la obesidad infantil.

Por otro lado el ítem cuantas horas dedicas actividad deportiva extraescolar, muestra la institución educativa "5 de Abril", los escolares practican 1 hora con un 40.8\% (127) y el 23.2\% (72) practican 2 horas, así mismo Institución “María Goretti” muestra que el 35.0\% (91) practican 2 horas y el 33.8\% (88) practican 3 horas. Por lo que quiere decir que aunque el porcentaje no sea mayor en las horas que dedicada actividad deportiva fuera del colegio, es importante destacar, a raíz del análisis de los resultados de nuestro estudio, que los escolares deberían fomentar el hábito saludable, realizar un mayor ejercicio físico, siendo necesario dirigirnos no solo a los escolares sino a sus padres, ya que a estas edades son ellos los que deciden, en las actividades de sus hijos. Según la Sanidad y el Ministerio de salud (2010) menciona que la actividad física debe continuar siendo una herramienta importante para promover la actividad y la condición física, generando un efecto positivo, más que negativo, sobre el rendimiento académico de los escolares.

La educación física relacionada con la salud se centra en el proceso encaminado a que el alumnado asuma progresivamente más responsabilidad en relación con su propia salud, su condición física y su bienestar.
Un estudio realizado por Calvo, et al. (2014) a través del test Krece plus en escolares de 8-12 años en Ecuador, mostró que el 38,3\% de los escolares dedica tan sólo 1-2 o ninguna hora a actividades deportivas extraescolares, concluyendo que los escolares deberían mejorar su estilo de vida, realizar educación para la salud y mayor actividad física.

Respecto a la actividad física según el género los rangos que más resaltan, están entre actividad física regular y mala, siendo que en la Institución 5 de abril tienen actividad física mala el 51.9\%(80) en el género masculino y el 50.3\%(79) el género femenino; así mismo actividad física regular el 45,5\% (70) género masculino y femenino el 44.6\% (70). De igual manera en la institución María Goretti la actividad física regular es 65.8\%(77) en el género masculino y el 70.6\%(101) en el género femenino, y más aún en la actividad física mala en el género masculino el 28.2\%(33) y en el género femenino el 25.2\%(36). Sin embargo se observa que no hay diferencias estadísticamente significativas en cuanto al género $(Z=-, 295: p>, 05)$ en ambas instituciones, no obstante los rangos que más resaltan son malo y regular .

De los Angeles y Rodriguez (2009) en su estudio en escolares de cuarto grado de primaria en la isla de Tenerife, encontraron que el 51,7\% tiene una puntuación regular, en el test el 39,3\% que corresponde a un $31,3 \%$ en las niñas y un $46,8 \%$ de los niños tienen una mala puntuación y solo el 13,2\% de las niñas y el 4,8\% de los niños tienen una buena puntuación. Mas aun Correa y Gutiérrez (2013) destacan que el $65,4 \%$ de las niñas frente al 38,1\% de los niños no presentan un nivel de actividad física bueno. Los resultados fueron similares al estudio, ya que el porcentaje de actividad física mala y regular predomina el género masculino. En la actividad física es importante hacer énfasis ya que a raíz de estos datos encontrados se puede ver que más de la mitad de los escolares del estudio deberían mejorar su estilo de vida, siendo necesario dirigirnos no solo a los escolares sino a sus padres quienes son los que educan a sus hijos en el hogar.

En otro estudio similar Calvo-Pacheco et al. (2014) mostraron que el $65 \%$ de las niñas y el 59,1\% de los niños no practican o practican ejercicios dos o menos horas de deporte a la semana. La media de horas de deporte extraescolar es de 2,2 para la totalidad de los escolares, sin diferencias por sexo a diferencia del estudio en Kid que afirman que en los niños del grupo de edad de 7 a 9 años, los varones hacen una media de 4,5 horas de deporte a la semana y las niñas 2,25 horas a la semana. 
En relación a la actividad física y el lugar de procedencia de los escolares se encontró que en la institución educativa pública " 5 de abril”, en la región sierra el 59.7\%(90) tienen actividad física mala, así mismo en la región selva es el 49.1,\%(26) y la región costa un 48.4\%(90) lo cual resalta que los escolares de la región sierra practican menos actividad física. Por otro lado la institución "María Goretti” se encontró en la región costa el 76,5\% (62) tienen actividad física regular, en la región sierra es un $69.2 \%$ (72) y la región selva es un 58.7\% (44), entonces los porcentajes reflejan que los escolares que menos practican actividad física son de la región costa. Sin embargo se observa que no hay diferencias estadísticamente significativas entre los puntajes alcanzados $\left(\chi^{2}=5,570 ;: \mathrm{p}>, 05\right)$ en la institución " 5 de abril”.

No obstante si se encontró diferencia estadísticamente significativa en la institución "María Goretti". En un estudio se mostró que el $40 \%$ de la población encuestada realiza poca actividad física (vida sedentaria), el $41.9 \%$ es de Lima metropolitana, el $46.9 \%$ el resto de la costa y el $36.8 \%$ de la selva, presentan escasa actividad física. Estos resultados estarían corroborando la tendencia hacia el sedentarismo en las zonas urbanizadas, en la que disminuye progresivamente la demanda del ejercicio físico (Ceballos, 2012).

\section{Comparación de variables entre las instituciones educativas públicas}

Considerando que las instituciones educativas desempeñan un importante papel en la formación integral de niños y niñas, se hace necesario identificar los hábitos alimentarios y actividad física en estos estudiantes, buscando comparar e identificar los factores para la aparición de problemas de salud, disminución del rendimiento físico y desempeño escolar en niños y niñas de un específico rango de edad (9 a 12 años) de las dos instituciones.

Al comparar los hábitos alimentarios entre las dos instituciones se encontró que existe diferencia ( $\mathrm{Z}=$ $-3,222 ; p<0.001)$ es decir, los hábitos alimentarios de los escolares en la institución educativa pública “María Goretti” el 77.7\%(202) presentaron hábitos alimentarios adecuados (altos) mientras que en la institución educativa "5 de abril” el 70.4\%(219) tienen hábitos alimentarios altos, resaltando el mayor porcentaje de escolares de la institución educativa "María Goretti” tienen mejor hábitos alimentarios, lo cual se debe a diferentes factores sociodemográficos, culturales y sociales como: La zona en la que se encuentran ubicadas las instituciones, el colegio
"5 de abril" se ubica en una zona autogestionaria de Huaycán, donde hay poca gente con nivel socioeconómico alto, hoy en día, cuenta con una población de aproximadamente 120000 habitantes, en una área de 576696 hectáreas. Está dividida en 26 zonas, de la zona A a la zona $\mathrm{Z}$, y sigue ampliándose con nuevas invasiones cada año. Los pobladores son mayormente de nivel socioeconómico bajo, obreros de manufactura, minas y construcción, o vendedores ambulantes. Las partes altas de Huaycán también se caracterizan por ser ciudades dormitorio: la mayoría de sus habitantes trabajan en Lima durante el día, y estas zonas quedan casi vacías hasta la noche. Durante el día, mientras los adultos trabajan, los niños y los ancianos se encuentran generalmente abandonados, expuestos a numerosos riesgos. Las zonas más necesitadas de Huaycán están ubicadas en las partes altas de los cerros: entre ellas, las zonas I, J, K, X, V,G y R donde interviene el Samusocial Perú. Son zonas de alto riesgo natural. (Instituto Nacional de Estadística e Informática, 2015).

A comparación de la institución "María Goretti" se ubica en una zona con nivel socioeconómico moderado. Por lo tanto el entorno social y económico predispone estos factores. Velasco y Costa (2008), refiere que en un Estudio Enkid 1998-2000, por Serra y col. (2000) demuestran que menos de la mitad de los jóvenes españoles con condiciones socioeconómico alto (4-24 años), tiene un buen nivel de alimentación. El resto presenta carencias o hábitos alimentarios inadecuados, y de éstos, el 4\% está muy lejos de seguir una alimentación adecuada.

Los hábitos alimentarios son infinitamente complejos, puesto que se derivan de las primeras experiencias del hombre y están influidos por numerosos factores tales como: la familia, el ambiente social, economía, geografía, región o religión. En general, estos factores son clasificados por distintos autores en: fisiológicos, físicos, familiares y sociales Serra y col. citado por (Velasco- y Costa, 2008).

Al comparar la actividad física de los escolares de las dos instituciones públicas se encontró que existe diferencias estadísticamente significativas entre los puntajes alcanzados $(Z=-6,707 ; p<, 000)$. Es decir los escolares de la institución educativa "5 de abril” dedican menos horas a la actividad física ya sea en el colegio o fuera del colegio, mientras que los escolares de la institución "María Goretti" realizan más actividad física. Se realizó un estudio en escolares de tercer grado de primaria en Santiago de Cali, con dos instituciones una pública y privada según Tafur,Idarraga y Ordoñez, (2015) mostró que el colegio Luis Fernando Caicedo con un 54\% y 
en los alumnos del Colombo Británico con un 64\% donde el tiempo que dedicaban a la actividad física era de 2 horas diarias, paralelo a lo establecido en la encuesta ENSIN 2010 donde se determinó que el $62 \%$ de los niños y adolescentes en Colombia ve TV o juega con videojuegos por dos o más horas diarias. Sin embargo, el hecho de ser instituciones públicas, no escapan de la realidad, al contrario si hay mucha diferencia, y como se menciona anteriormente el nivel socioeconómico, es un factor de gran influencia, si bien es cierto para practicar actividad física no depende el tipo de institución, sin embargo sí depende, del tipo de educación en salud y de la práctica de los hábitos aprendidos ya sea en el hogar o dentro de la misma institución por parte de los docentes.

\section{Declaración de financiamiento y de conflicto de intereses:}

El estudio fue financiado por los autores, quienes declaran no tener algún tipo de conflicto de interés en la investigación realizada.

\section{Correspondencia:}

Naara Andrea Hernández Oblitas

Licenciada en Enfermería. Clínica Good Hope, Malecón Balta 956, Miraflores, Lima, Perú e-mail: naara.hernandez@upeu.edu.pe

\section{REFERENCIAS BIBLIOGRÁFICAS}

Andre'. Toschke, Helmut Ku, † Berthold Koletzko, R. von K. (2005). Meal Frequency and Childhood Obesity. Diet and Physical Activity.

Arboccó, M., \& Arboccó, J. O. B. (2012). Impacto de la “ televisión basura " en la mente y la conducta de niños y adolescentes Impact of " trash TV” on children and adolescents " mind and behavior. Avances En Psicología, 20(2), 43-57.

Barbancho Cisneros, F. J. (2005). Efectos de la televisión sobre la actividad física y el rendimiento escolar en niñas escolares. Cultura de Los Cuidados, 17, 88-93. Retrieved from http://rua.ua.es/dspace/ bitstream/10045/1014/1/culturacuidados_17_14.pdf

Bayona, I. (2013). Promoción de hábitos alimentarios saludables desde la escuela.

Calle, Montaleza, M. (2011). valoración del bajo peso, sobrepeso y obesidad en los niños/as de 2do a $7 \mathrm{mo}$ de educación básica de la escuela fiscal Emilio Murillo de la comunidad de San Pedro del Cebollar cuenca, ecuador 2010-2011. El Escorial, (Imc), 34,56.

Calvo-pacheco, M., Rodríguez-álvarez, C., Abreu, R., Aguirre-jaime, A., \& Arias, Á. (2014). Valoración del estado nutricional de escolares de primaria de la isla de Tenerife (España) Assessment of nutritional status of primary school children IN. Hig. Sanid. Ambient., 14(1), 1171-1177.

Carbajal y Pinto. (2015). nutrición y salud. The Effects of Brief Mindfulness Intervention on Acute Pain Experience: An Examination of Individual Difference, 1, 1689-1699. http://doi.org/10.1017/ CBO9781107415324.004

Castañeda, O., Rocha, J., \& Ramos, M. (2008). Evaluación de los hábitos alimenticios y estado nutricional en adolescentes de Sonora. Archivos de Medicina Familiar, 10(hábitos alimenticios 9 sonora)), 7-9.
Ceballos, O. (2012). Actividad Física en el Adulto Mayor.

Correa , Gutiérrez, M. . (2013). Hábitos alimentarios y de actividad física en escolares de la provincia de Granada. Nure Investigation, 67(1), 1-8.

De los angeles A., Rodriguez C., R. A. (2009). Estudio antropométrico y educación nutricional en escolares de la isla de Tenerife.

EROSKI, F. (2015). Importancia del desayuno. Idea Sana EROSKI.

Espino, L. H., Valladares, C., \& Satalaya, A. (2004). Estado Nutricional y Factores culturales que influyen en los hábitos alimentarios . Estudio en población Ashaninka y Aguaruna. Instituto Nacional de Salud, 1-27.

Espinosa, campoverde y. (2015). Jenny Alexandra Campoverde Ayora Paula Belén Espinoza Berrezueta $1,1-71$.

Funes, B., \& M., G. (2010). Características Alimentarias De Los Escolares De 6 a 12 Años Con Sobrepeso Y Obesidad De La Escuela No 215 Octavia Ricardone. Bigand, Provincia De Santa Fe.

Guerrero, I. y L. (2005). factores que intervienen en la modificación de hábitos alimentarios no adecuados en la provincia de palena, I, 1-12.

Llargués, E., Franco, R., Recasens, A., Nadal, A., Vila, M., José Pérez, M., ... Castells, C. (2009). Estado ponderal, hábitos alimentarios y de actividad física en escolares de primer curso de educación primaria: estudio AVall. Endocrinologia y Nutricion, 56(6), 287-292. http://doi. org/10.1016/S1575-0922(09)71943-6

Macias, A., Gordillo, L., \& Camacho, E. (2012). Habitos alimentarios en niños en edad escolar y el papel de la educación para la salud.pdf. Rev Chil Nutr, 39(3), 4043. http://doi.org/10.4067/S0717-75182012000300006 
Macias M, A. I., Gordillo S, L. G., \& Camacho R, E. J. (2012). Hábitos alimentarios de niños en edad escolar y el papel de la educación para la salud. Revista Chilena de Nutrición, 39(3), 40-43. http://doi.org/10.4067/ S0717-75182012000300006

Marin-Zegarra, K., Olivares-Atahualpa, S., Solano-Isidro, P., \& Musablon-Oyitas, Y. (2011). Estado nutricional y hábitos alimentarios de los alumnos del nivel secundaria de un colegio nacional. Revista de Enfermería Herediana, 4(2), 64-70. Retrieved from http://www. upch.edu.pe/faenf/images/stories/articulorevista4/ estado nutricional3

Martínez, Á. E., Gomis, I. M., Moraga, A. B., Ferrer, C., Fábrega, M. T., \& Bueno, Á. F. (2010). Estilos de vida , hábitos dietéticos y prevalencia del sobrepeso y la obesidad en una población infantil, XII, 53-65.

OMS. (2015). OMS | Alimentación sana. Alimentación Sana. Retrieved from http://www.who.int/mediacentre/ factsheets/fs394/es/\#.Vo7BGZkhL14.mendeley

Orgiles, M., Sanz, I., Piqueras, J. A., \& Espada, J. P. (2014). [Differences in eating habits and physical activity in a sample of preadolescent depending on their weight category]. Nutricion Hospitalaria, 30(2), 306-313. http://doi.org/10.3305/nh.2014.30.2.7607

Peña, M., \& Bacallao, J. (2001). La obesidad y sus tendencias en la Región. Revista Panamericana de Salud Pública, 10(2), 45-78. http://doi.org/10.1590/ S1020-49892001000800001

Rojas, A., León, M., \& Sánchez, O. (2003). Formación de hábitos alimentarios y de estilos de vida saludables. Curriculum de Educación Inicial, 1-36. Retrieved from http://www.unicef.org/venezuela/spanish/educinic9. pdf
Román, V., \& Quintana, M. (2010). La alimentación saludable en adolescentes de colegios públicos de un distrito de Lima Media influence on adolescents' healthy nutrition in Lima district public schools. An Fac Med., 71(3), 185-189. Retrieved from www.elseiver.es

Sanidad, C. M. de E. y M. de. (2010). La Actividad Física en el entorno escolar. Actividad física y salud en la infancia y la adolescencia. Guía para todas las personas que participan en su educación., 73-82.

Serafin, P. (2012). Manual de la alimentación escolar saludable. Instituto Nacional de Alimentación y Nutrición, 1-78.

Tafur, Diaz, Mauricio, O. y O. (2015). Hábitos alimentarios y nivel de actividad física de los estudiantes de tercero a séptimo grado de dos colegios de santiago de cali: colegio Luis Fernando Caicedo y colegio colombo británico. The Effects of Brief Mindfulness Intervention on Acute Pain Experience: An Examination of Individual Difference, 1, 1689-1699. http://doi. org/10.1017/CBO9781107415324.004

Velasco-Costa, J. (2008). Evaluación de la dieta en escolares de Granada, 259.

Zegarra, M., Olivares-Atahualpa, K., Solano-Isidro, S. ., \& Musablon-Oyitas, P. (2011). Estado nutricional y hábitos alimentarios de los alumnos del nivel secundaria de un colegio nacional. Revista de Enfermería Herediana, 4(2), 64-70. Retrieved from http://www. upch.edu.pe/faenf/images/stories/articulorevista4/ estado nutricional3

Recibido: 14/04/2015 Aceptado: 25/08/2015 